\title{
Effect of different storage conditions and seed treatments on seed viability in soybean [Glycine max (L.) Merr.]
}

\author{
J. B. Patel*, Jyoti Sondarva, C. A. Babariya, R. R. Rathod and V. J. Bhatiya \\ Department of Seed Science and Technology, Junagadh Agricultural University, Junagadh -362001(Gujarat), INDIA \\ *Corresponding author. E-mail: jbpatelvasai38@gmail.com
}

Received: July 9, 2016; Revised received: October 26, 2016; Accepted: January 25, 2017

\begin{abstract}
The present investigation was carried out in laboratory of the Department of Seed Science and Technology, College of Agriculture, Junagadh Agricultural University, Junagadh from the April 2013 to April 2015, wherein two $\mathrm{kg}$ of freshly harvested quality seed of soybean cv. Gujarat Junagadh Soybean 3 having high germination percentage and low moisture content (below 8\%) was taken for each repetition and for each combination of treatments. The treatment consisted of two storage conditions (C) viz., $\mathrm{C}_{1}$ (Ambient temperature) and $\mathrm{C}_{2}$ (Cold storage at $7^{\circ} \mathrm{C} \pm 2^{\circ} \mathrm{C}$ ), and five seed treatments (S) viz., $S_{1}=$ Control, $S_{2}=$ Carbendazim @ $2 \mathrm{~g} / \mathrm{kg}$ seed, $S_{3}=$ Mancozeb @ $2 \mathrm{~g} /$ $\mathrm{kg}$ seed, $S_{4}=$ Neem leaf powder @ 10g/kg seed, and $S_{5}=$ Neem Oil @ $5 \mathrm{ml} / \mathrm{kg}$ seed. The experiment was carried out using Completely Randomized Design (Factorial) repeated three times. After proper mixing or smearing the seeds as per the treatments, seeds were packed in cloth bag and kept in laboratory under two different storage conditions. Observations were recorded at 90 days interval on germination $(\%)$, root length $(\mathrm{cm})$, shoot length $(\mathrm{cm})$, seedling dry weight $(\mathrm{g})$, seed vigour index I, seed vigour index II and seed moisture content (\%). The results revealed that storage condition (C) and seed treatments (S) exhibited significant differences almost for the all the traits for germination and seedling parameters after 2 years of storage. The results of soybean seed stored in two different storage conditions showed that on an average, the seed stored under cold storage $\left(7^{0} \mathrm{C} \pm 2^{\circ} \mathrm{C}\right)$ noted higher values for all the traits studied except seed moisture content after 2 years of storage. Among the seed treatments, on an average, after 2 years of seed storage, significantly $(P<0.05)$ higher values were recorded by all the seed treatments over the control. However, seed treated with Mancozeb @ $2 \mathrm{~g} / \mathrm{kg}$ of seed recorded the significantly highest germination percentage $(71.50 \%)$ and it was at par with Neem leaf powder @ $10 \mathrm{~g} / \mathrm{kg}$ seed $(70.67 \%)$ and Carbendazim @ $2 \mathrm{~g} / \mathrm{kg}$ seed $(69.67 \%)$ after 2 years of storage. The germination percentage noted in control treatment was 33.17 per cent after 2 years of storage. An ISTA standard for germination in soybean is 70 per cent. Most of the interactions effects were found significant $(P<0.05)$ for all the traits studied.
\end{abstract}

Keywords: Seed treatment, Soybean, Storage condition, Viability

\section{INTRODUCTION}

Soybean (Glycine max (L.) Merr) is an important oilseed crop, is listed as poor storer. It loses viability rapidly under warm and humid storage conditions. One of the major constraints in soybean cultivation is the non-availability of high vigour seeds at the time of sowing. Now-a-days, the area and production of this crop is increasing gradually, but productivity remains almost constant (Mahesh Babu and Hunje, 2008). Poor seed germination is a major constraint for increasing the productivity of soybean.

High quality seed that provides adequate plant stand is the basis for profitable production and expansion of soybean crop. In order to increase the production of soybean, a source of high quality, disease free seed must be established and maintained. Loss of viability and vigour under high temperature and relative humidity conditions is a common phenomenon in many crop seeds but it is well marked in soybean. Under adverse conditions such as the temperature above $30^{\circ} \mathrm{C}$ and relative air humidity from 80 to 90 per cent, the variation in seed germination rate can be high. It seems that temperature, moisture and storage duration are the most important individual factors which affected the stored product quality and quantity (Sisman, 2005). Fabrizius et al. (1999) confirmed the possibility of predicting the actual germination rate of soybean seed during natural aging by applying the accelerated aging test, the main factors being the time of natural aging duration and degree of seed deterioration.

Many of synthetic chemicals look effective, but they are not readily degradable physically or biologically which yield more toxic residues. Hence, the feasible approach is the treatment of seeds with botanicals which are safe, economical, eco-friendly, cheap, easily locally available and non-harmful to seeds, animals and human beings. It will be of immense use to the farming community. Therefore, the present investigation was carried out to know how the soybean seed can be stored by treating them with fungicides or botanicals under specific storage conditions for longer period 
with minimum qualitative and quantitative changes.

\section{MATERIALS AND METHODS}

The present investigation was carried out in laboratory of the Department of Seed Science and Technology, College of Agriculture, Junagadh Agricultural University, Junagadh from the April 2013 to April 2015, wherein two $\mathrm{kg}$ of freshly harvested quality seed of soybean cv. GJS 3 having high germination percentage and low moisture content (below 8\%) was taken for each repetition and for each combination of treatments. The treatment consisted of two storage conditions (C) viz., $\mathrm{C}_{1}$ (Ambient temperature) and $\mathrm{C}_{2}$ (Cold storage at $7^{\circ} \mathrm{C} \pm 2^{\circ} \mathrm{C}$ ), and five seed treatments (S) viz., $\mathrm{S}_{1}=\mathrm{Con}-$ trol, $\mathrm{S}_{2}=$ Carbendazim @ $2 \mathrm{~g} / \mathrm{kg}$ seed, $\mathrm{S}_{3}=$ Mancozeb (a) 2g/kg seed, $\mathrm{S}_{4}=$ Neem leaf powder @ 10g/kg seed, and $\mathrm{S}_{5}=$ Neem Oil @ $5 \mathrm{ml} / \mathrm{kg}$ seed. The experiment was carried out using Completely Randomized Design (Factorial) repeated three times. After proper mixing or smearing the seeds as per the treatments, seeds were packed in muslin cloth bag and kept in laboratory under two different storage conditions. Observations were recorded at 90 days interval on germination (\%), root length $(\mathrm{cm})$, shoot length $(\mathrm{cm})$, seedling dry weight $(\mathrm{g})$, seed vigour index I, seed vigour index II and seed moisture content (\%).Germination test was carried out using paper towel technique as per the procedure given by ISTA (1999). Germinated seeds were counted on $8^{\text {th }}$ day and 10 germinated seedlings were selected from each replication of the treatment for cal- culating the seedling vigour index. The seedling vigour index (length and dry weight) was calculated as per the formula suggested by Abdul-Baki and Anderson, 1973). The shoot and root length of each of the 10 seedlings were measured in centimeters. Seedling dry weight was measured of all the germinated seedlings after oven drying. Seed moisture content was determined by oven dry method. The data were statistically analyzed as per the method of Cochran and Cox (1957) for Completely Randomized Design (Factorial).

\section{RESULTS AND DISCUSSION}

The results presented in Table 1 revealed that storage condition (C) and seed treatments (S) exhibited significant differences for germination per cent under laboratory in soybean for almost all the dates of recording observations and after 2 years of storage. The data of soybean seed stored under two storage conditions revealed that seed stored under cold storage $\left(7^{\circ} \mathrm{C} \pm 2^{\circ} \mathrm{C}\right)$ noted significantly higher germination $(76.13 \%)$ after the period of 2 years of storage. Among the seed treatments, seed treated with Mancozeb @ $2 \mathrm{~g} / \mathrm{kg}$ of seed recorded the significantly highest germination percentage $(71.50 \%)$ and it was at par with Neem leaf powder@10g/kg seed (70.67\%) and Carbendazim @ $2 \mathrm{~g} / \mathrm{kg}$ seed $(69.67 \%)$ after 2 years of storage. The germination percentage noted in control treatment was 33.17 per cent after 2 years of storage stored under ambient condition. An ISTA standard for germination is 70 per cent for soybean. Over all the treatment

Table 1. Effects of storage condition and various seed treatments on germination in soybean.

\begin{tabular}{|c|c|c|c|c|c|c|c|c|}
\hline Factor/Period & 3 Month & 6 Month & 9 Month & 12 Month & 15 Month & 18 Month & 21 Month & 24 Month \\
\hline $\mathrm{C}_{1}$ & 96.20 & 94.20 & 94.33 & 89.93 & 82.33 & 75.67 & 63.66 & 48.20 \\
\hline $\mathrm{C}_{2}$ & 95.47 & 88.20 & 95.33 & 90.40 & 89.53 & 90.07 & 85.53 & 76.13 \\
\hline Mean & 95.83 & 91.20 & 94.83 & 90.17 & 85.93 & 82.87 & 74.60 & 62.16 \\
\hline S. Em \pm & 0.30 & 0.57 & 0.15 & 0.64 & 0.46 & 0.55 & 0.42 & 0.54 \\
\hline C.D. at $5 \%$ & NS & 1.70 & 0.44 & NS & 1.37 & 1.63 & 1.25 & 1.61 \\
\hline $\mathrm{S}_{1}$ & 95.00 & 89.83 & 94.83 & 91.00 & 78.17 & 72.00 & 61.50 & 33.17 \\
\hline $\mathrm{S}_{2}$ & 96.67 & 89.50 & 95.00 & 88.83 & 87.17 & 85.33 & 77.17 & 69.67 \\
\hline $\mathrm{S}_{3}$ & 96.00 & 92.83 & 95.00 & 90.17 & 88.83 & 86.83 & 79.17 & 71.50 \\
\hline $\mathrm{S}_{4}$ & 94.67 & 91.33 & 93.17 & 89.17 & 88.00 & 84.33 & 79.17 & 70.67 \\
\hline $\mathrm{S}_{5}$ & 96.83 & 92.50 & 96.17 & 91.67 & 87.50 & 85.83 & 76.00 & 63.83 \\
\hline Mean & 95.83 & 91.20 & 94.83 & 90.17 & 85.93 & 82.87 & 74.60 & 62.16 \\
\hline S. Em + & 0.48 & 0.91 & 0.24 & 1.01 & 0.73 & 0.87 & 0.66 & 0.86 \\
\hline C.D. at $5 \%$ & 1.42 & NS & 0.70 & NS & 5.16 & 2.58 & 1.97 & 2.55 \\
\hline $\mathrm{C}_{1 \times} \mathrm{S}_{1}$ & 95.67 & 93.33 & 95.00 & 93.33 & 76.00 & 68.33 & 57.33 & 33.00 \\
\hline $\mathrm{C}_{1 \times} \mathrm{S}_{2}$ & 98.00 & 93.33 & 94.67 & 88.00 & 84.33 & 78.00 & 65.00 & 54.00 \\
\hline $\mathrm{C}_{1 \times} \mathrm{S}_{3}$ & 95.67 & 95.33 & 96.67 & 89.00 & 85.67 & 78.33 & 65.00 & 55.00 \\
\hline $\mathrm{C}_{1 \times} \mathrm{S}_{4}$ & 94.67 & 94.33 & 90.00 & 88.67 & 82.67 & 76.00 & 64.33 & 55.00 \\
\hline $\mathrm{C}_{1 \times} \mathrm{S}_{5}$ & 97.00 & 94.67 & 95.33 & 90.67 & 83.00 & 77.67 & 66.67 & 44.00 \\
\hline $\mathrm{C}_{2 \times} \mathrm{S}_{1}$ & 94.33 & 86.33 & 94.67 & 88.67 & 80.33 & 75.67 & 65.67 & 37.33 \\
\hline $\mathrm{C}_{2 \times} \mathrm{S}_{2}$ & 95.33 & 85.67 & 95.33 & 89.67 & 90.00 & 92.67 & 89.33 & 85.33 \\
\hline $\mathrm{C}_{2 \times} \mathrm{S}_{3}$ & 96.33 & 90.33 & 93.33 & 91.33 & 92.00 & 95.33 & 93.33 & 88.00 \\
\hline $\mathrm{C}_{2 \times} \mathrm{S}_{4}$ & 94.67 & 88.33 & 96.33 & 89.67 & 93.33 & 92.67 & 94.00 & 86.33 \\
\hline $\mathrm{C}_{2 \times} \mathrm{S}_{5}$ & 96.67 & 90.33 & 97.00 & 92.67 & 92.00 & 94.00 & 85.33 & 83.67 \\
\hline Mean & 95.83 & 91.20 & 94.83 & 90.17 & 85.93 & 82.87 & 74.60 & 62.16 \\
\hline S. Em + & 0.68 & 1.28 & 0.33 & 1.43 & 1.03 & 1.23 & 0.94 & 1.21 \\
\hline C.D. at $5 \%$ & NS & NS & 0.99 & NS & 3.05 & 3.65 & 2.78 & 3.61 \\
\hline $\mathrm{CV} \%$ & 1.22 & 2.44 & 0.61 & 2.75 & 2.07 & 2.57 & 2.18 & 3.39 \\
\hline
\end{tabular}


J. B. Patel et al. / J. Appl. \& Nat. Sci. 9 (1): 245- 252 (2017)

Table 2. Effects of storage condition and various seed treatments on seedling root length in soybean.

\begin{tabular}{|c|c|c|c|c|c|c|c|c|}
\hline Factor/Period & 3 Month & 6 Month & 9 Month & 12 Month & 15 Month & 18 Month & 21 Month & 24 Month \\
\hline $\mathrm{C}_{1}$ & 8.74 & 6.69 & 9.00 & 8.90 & 8.59 & 9.36 & 5.49 & 5.49 \\
\hline $\mathrm{C}_{2}$ & 9.36 & 6.39 & 8.07 & 8.13 & 7.77 & 10.90 & 5.48 & 5.40 \\
\hline Mean & 9.05 & 6.54 & 8.53 & 8.51 & 8.18 & 10.13 & 5.49 & 5.45 \\
\hline S. Em \pm & 0.36 & 0.15 & 0.10 & 0.10 & 0.19 & 0.20 & 0.13 & 0.12 \\
\hline C.D. at $5 \%$ & NS & NS & 0.31 & 0.29 & 0.56 & 0.60 & NS & NS \\
\hline $\mathrm{S}_{1}$ & 10.25 & 6.47 & 9.48 & 9.33 & 7.54 & 9.96 & 5.81 & 6.00 \\
\hline $\mathrm{S}_{2}$ & 9.52 & 6.61 & 9.10 & 8.89 & 8.46 & 9.70 & 5.24 & 5.16 \\
\hline $\mathrm{S}_{3}$ & 9.61 & 6.39 & 8.32 & 8.48 & 7.34 & 10.51 & 5.89 & 5.71 \\
\hline $\mathrm{S}_{4}$ & 8.56 & 6.53 & 7.54 & 7.69 & 8.53 & 9.72 & 5.26 & 5.22 \\
\hline $\mathrm{S}_{5}$ & 7.33 & 6.70 & 8.23 & 8.17 & 9.04 & 10.77 & 5.23 & 5.14 \\
\hline Mean & 9.05 & 6.54 & 8.53 & 8.51 & 8.18 & 10.13 & 5.49 & 5.45 \\
\hline S. Em \pm & 0.56 & 0.23 & 0.16 & 0.16 & 0.30 & 0.32 & 0.21 & 0.19 \\
\hline C.D. at $5 \%$ & 1.67 & NS & 0.48 & 0.46 & 0.89 & NS & NS & 0.56 \\
\hline $\mathrm{C}_{1 \mathrm{X}} \mathrm{S}_{1}$ & 11.31 & 6.45 & 9.30 & 9.05 & 7.88 & 8.81 & 5.73 & 6.14 \\
\hline $\mathrm{C}_{1 \times} \mathrm{S}_{2}$ & 9.54 & 6.68 & 9.21 & 8.87 & 8.76 & 9.13 & 5.22 & 5.19 \\
\hline $\mathrm{C}_{1 \times} \mathrm{S}_{3}$ & 9.40 & 6.96 & 9.03 & 9.30 & 8.59 & 10.13 & 5.86 & 5.64 \\
\hline $\mathrm{C}_{1 \times} \mathrm{S}_{4}$ & 8.33 & 6.54 & 8.65 & 8.51 & 8.84 & 8.85 & 5.29 & 5.21 \\
\hline $\mathrm{C}_{1 \mathrm{X}} \mathrm{S}_{5}$ & 5.20 & 6.84 & 8.85 & 8.77 & 8.89 & 9.86 & 5.36 & 5.29 \\
\hline $\mathrm{C}_{2 \times} \mathrm{S}_{1}$ & 9.18 & 6.48 & 9.66 & 9.60 & 7.19 & 11.10 & 5.89 & 5.85 \\
\hline $\mathrm{C}_{2 \times} \mathrm{S}_{2}$ & 9.50 & 6.55 & 8.98 & 8.91 & 8.16 & 10.26 & 5.25 & 5.16 \\
\hline $\mathrm{C}_{2 \times} \mathrm{S}_{3}$ & 9.88 & 5.88 & 7.61 & 7.67 & 6.09 & 10.89 & 5.92 & 5.77 \\
\hline $\mathrm{C}_{2 \times} \mathrm{S}_{4}$ & 8.78 & 6.53 & 6.44 & 6.88 & 8.22 & 10.59 & 5.23 & 5.23 \\
\hline $\mathrm{C}_{2 \times} \mathrm{S}_{5}$ & 9.47 & 6.57 & 7.60 & 7.57 & 9.19 & 11.68 & 5.10 & 5.00 \\
\hline Mean & 9.05 & 6.54 & 8.53 & 8.51 & 8.18 & 10.13 & 5.49 & 5.45 \\
\hline S. Em \pm & 0.80 & 0.33 & 0.23 & 0.22 & 0.42 & 0.45 & 0.30 & 0.27 \\
\hline C.D. at $5 \%$ & 2.36 & NS & 0.68 & 0.65 & 1.26 & NS & NS & NS \\
\hline $\mathrm{CV} \%$ & 15.21 & 8.72 & 4.65 & 4.45 & 8.99 & 7.69 & 9.41 & 8.45 \\
\hline
\end{tabular}

Table 3. Effects of storage condition and various seed treatments on seedling shoot length in soybean.

\begin{tabular}{|c|c|c|c|c|c|c|c|c|}
\hline Factor/Period & 3 Month & 6 Month & 9 Month & 12 Month & 15 Month & 18 Month & 21 Month & 24 Month \\
\hline $\mathrm{C}_{1}$ & 5.16 & 3.36 & 4.13 & 4.70 & 4.30 & 5.78 & 4.60 & 4.49 \\
\hline $\mathrm{C}_{2}$ & 5.39 & 3.55 & 3.40 & 4.41 & 3.70 & 6.45 & 5.29 & 5.37 \\
\hline Mean & 5.27 & 3.45 & 3.76 & 4.56 & 4.00 & 6.11 & 4.95 & 4.93 \\
\hline S. Em \pm & 0.19 & 0.23 & 0.05 & 0.14 & 0.12 & 0.15 & 0.14 & 0.10 \\
\hline C.D. at $5 \%$ & NS & NS & 0.15 & NS & 0.37 & 0.43 & 0.40 & 0.29 \\
\hline $\mathrm{S}_{1}$ & 5.85 & 3.18 & 3.99 & 4.88 & 3.76 & 6.64 & 4.59 & 4.59 \\
\hline $\mathrm{S}_{2}$ & 5.76 & 3.28 & 3.93 & 4.78 & 3.87 & 5.93 & 4.82 & 5.02 \\
\hline $\mathrm{S}_{3}$ & 5.28 & 3.95 & 3.68 & 4.37 & 4.24 & 5.88 & 4.69 & 4.65 \\
\hline $\mathrm{S}_{4}$ & 5.35 & 3.21 & 3.63 & 4.34 & 4.03 & 5.89 & 5.14 & 5.23 \\
\hline $\mathrm{S}_{5}$ & 4.12 & 3.63 & 3.58 & 4.41 & 4.07 & 6.23 & 5.49 & 5.16 \\
\hline Mean & 5.27 & 3.45 & 3.76 & 4.56 & 4.00 & 6.11 & 4.95 & 4.93 \\
\hline $\mathrm{S} . \mathrm{Em} \pm$ & 0.29 & 0.36 & 0.08 & 0.21 & 0.20 & 0.23 & 0.21 & 0.16 \\
\hline C.D. at $5 \%$ & 0.87 & NS & 0.24 & NS & NS & NS & 0.64 & 0.46 \\
\hline $\mathrm{C}_{1 \times} \mathrm{S}_{1}$ & 5.92 & 3.02 & 4.14 & 4.86 & 4.05 & 6.20 & 4.30 & 4.26 \\
\hline $\mathrm{C}_{1 \times} \mathrm{S}_{2}$ & 6.16 & 3.28 & 4.09 & 4.76 & 3.94 & 5.34 & 4.50 & 4.62 \\
\hline $\mathrm{C}_{1 \times} \mathrm{S}_{3}$ & 5.55 & 3.53 & 4.03 & 4.54 & 5.01 & 5.45 & 4.59 & 4.30 \\
\hline $\mathrm{C}_{1 \times} \mathrm{S}_{4}$ & 5.29 & 3.36 & 4.08 & 4.57 & 4.24 & 5.78 & 5.19 & 5.07 \\
\hline $\mathrm{C}_{1 \times} \mathrm{S}_{5}$ & 2.86 & 3.82 & 4.11 & 4.77 & 4.24 & 6.12 & 4.43 & 4.23 \\
\hline $\mathrm{C}_{2 \times} \mathrm{S}_{1}$ & 5.79 & 3.34 & 3.84 & 4.90 & 3.47 & 7.08 & 4.88 & 4.91 \\
\hline $\mathrm{C}_{2 \times} \mathrm{S}_{2}$ & 5.26 & 3.28 & 3.77 & 4.81 & 3.81 & 6.52 & 5.13 & 5.41 \\
\hline $\mathrm{C}_{2 \times} \mathrm{S}_{3}$ & 5.01 & 4.37 & 3.23 & 4.20 & 3.47 & 6.30 & 4.79 & 5.00 \\
\hline $\mathrm{C}_{2 \times} \mathrm{S}_{4}$ & 5.41 & 3.30 & 2.99 & 4.11 & 3.82 & 5.99 & 5.08 & 5.40 \\
\hline $\mathrm{C}_{2 \times} \mathrm{S}_{5}$ & 5.36 & 3.44 & 3.05 & 4.04 & 3.91 & 6.34 & 6.56 & 6.10 \\
\hline Mean & 5.27 & 3.45 & 3.76 & 4.56 & 4.00 & 6.11 & 4.95 & 4.93 \\
\hline S. Em \pm & 0.12 & 0.50 & 0.12 & 0.30 & 0.28 & 0.33 & 0.30 & 0.22 \\
\hline C.D. at $5 \%$ & 0.41 & NS & 0.34 & NS & NS & NS & 0.90 & 0.66 \\
\hline $\mathrm{CV} \%$ & 13.61 & 15.14 & 5.35 & 11.53 & 11.93 & 9.25 & 10.62 & 7.77 \\
\hline
\end{tabular}


J. B. Patel et al. / J. Appl. \& Nat. Sci. 9 (1): 245- 252 (2017)

Table 4. Effects of storage condition and various seed treatments on seedling dry weight in soybean.

\begin{tabular}{|c|c|c|c|c|c|c|c|c|}
\hline Factor/Period & 3 Month & 6 Month & 9 Month & 12 Month & 15 Month & 18 Month & 21 Month & 24 Month \\
\hline $\mathrm{C}_{1}$ & 8.81 & 9.65 & 9.79 & 8.48 & 8.84 & 9.42 & 4.53 & 4.48 \\
\hline $\mathrm{C}_{2}$ & 8.74 & 10.05 & 10.19 & 8.57 & 9.49 & 8.89 & 4.47 & 4.37 \\
\hline Mean & 8.77 & 9.85 & 9.99 & 8.52 & 9.16 & 9.16 & 4.50 & 4.42 \\
\hline S. Em \pm & 0.10 & 0.11 & 0.11 & 0.12 & 0.11 & 0.11 & 0.04 & 0.05 \\
\hline C.D. at $5 \%$ & NS & 0.33 & 0.34 & NS & 0.34 & 0.34 & NS & NS \\
\hline $\mathrm{S}_{1}$ & 8.97 & 9.31 & 9.73 & 9.00 & 8.81 & 9.14 & 4.73 & 4.60 \\
\hline $\mathrm{S}_{2}$ & 8.73 & 9.74 & 10.10 & 9.00 & 8.95 & 8.94 & 4.60 & 4.62 \\
\hline $\mathrm{S}_{3}$ & 8.98 & 10.23 & 10.43 & 8.53 & 9.33 & 9.57 & 4.39 & 4.28 \\
\hline $\mathrm{S}_{4}$ & 8.31 & 9.87 & 9.63 & 8.12 & 9.48 & 9.00 & 4.38 & 4.25 \\
\hline $\mathrm{S}_{5}$ & 8.88 & 10.10 & 10.04 & 7.97 & 9.24 & 9.13 & 4.42 & 4.37 \\
\hline Mean & 8.77 & 9.85 & 9.99 & 8.52 & 9.16 & 9.16 & 4.50 & 4.42 \\
\hline S. Em \pm & 0.17 & 0.18 & 0.18 & 0.19 & 0.18 & 0.18 & 0.06 & 0.08 \\
\hline C.D. at $5 \%$ & NS & 0.53 & 0.53 & 0.56 & NS & NS & 0.16 & 0.23 \\
\hline $\mathrm{C}_{1 \times} \mathrm{S}_{1}$ & 9.08 & 9.13 & 10.03 & 8.78 & 8.57 & 9.85 & 4.73 & 4.63 \\
\hline $\mathrm{C}_{1 \times} \mathrm{S}_{2}$ & 9.23 & 9.15 & 9.73 & 9.09 & 8.61 & 9.14 & 4.61 & 4.69 \\
\hline $\mathrm{C}_{1 \times} \mathrm{S}_{3}$ & 8.55 & 10.60 & 10.46 & 8.52 & 8.97 & 10.01 & 4.39 & 4.21 \\
\hline $\mathrm{C}_{1 \times} \mathrm{S}_{4}$ & 8.29 & 9.52 & 9.01 & 8.22 & 8.74 & 9.27 & 4.28 & 4.22 \\
\hline $\mathrm{C}_{1 \times} \mathrm{S}_{5}$ & 8.87 & 9.89 & 9.76 & 7.77 & 9.30 & 8.84 & 4.63 & 4.64 \\
\hline $\mathrm{C}_{2 \times} \mathrm{S}_{1}$ & 8.86 & 9.52 & 9.46 & 9.22 & 9.04 & 8.42 & 4.72 & 4.57 \\
\hline $\mathrm{C}_{2 \times} \mathrm{S}_{2}$ & 8.22 & 10.38 & 10.53 & 8.92 & 9.28 & 8.75 & 4.59 & 4.55 \\
\hline $\mathrm{C}_{2 \times} \mathrm{S}_{3}$ & 9.41 & 9.95 & 10.42 & 8.54 & 9.69 & 9.13 & 4.38 & 4.35 \\
\hline $\mathrm{C}_{2 \times} \mathrm{S}_{4}$ & 8.33 & 10.31 & 10.28 & 8.02 & 10.23 & 8.74 & 4.47 & 4.29 \\
\hline $\mathrm{C}_{2 \times} \mathrm{S}_{5}$ & 8.89 & 10.35 & 10.36 & 8.16 & 9.18 & 9.42 & 4.20 & 4.10 \\
\hline Mean & 8.77 & 9.87 & 10.04 & 8.52 & 9.16 & 9.16 & 4.50 & 4.42 \\
\hline S. Em \pm & 0.23 & 0.25 & 0.25 & 0.26 & 0.25 & 0.26 & 0.08 & 0.11 \\
\hline C.D. at $5 \%$ & 0.69 & 0.75 & 0.75 & NS & NS & 0.76 & 0.23 & 0.32 \\
\hline CV \% & 4.61 & 4.40 & 4.38 & 5.37 & 4.80 & 4.80 & 2.98 & 4.25 \\
\hline
\end{tabular}

Table 5. Effects of storage condition and various seed treatments on seed vigour index-I in soybean.

\begin{tabular}{|c|c|c|c|c|c|c|c|c|}
\hline Factor/Period & 3 Month & 6 Month & 9 Month & 12 Month & 15 Month & 18 Month & 21 Month & 24 Month \\
\hline $\mathrm{C}_{1}$ & 1336.68 & 947.18 & 1236.04 & 1223.24 & 1044.97 & 1187.04 & 801.41 & 480.57 \\
\hline $\mathrm{C}_{2}$ & 1407.99 & 876.08 & 1092.46 & 1126.83 & 975.59 & 1453.25 & 854.09 & 819.40 \\
\hline Mean & 1372.33 & 911.63 & 1164.25 & 1175.03 & 1010.28 & 1320.14 & 827.75 & 649.98 \\
\hline S. Em + & 46.79 & 17.33 & 11.98 & 17.94 & 20.70 & 22.65 & 21.09 & 11.42 \\
\hline C.D. at $5 \%$ & NS & 51.48 & 35.59 & 53.29 & 61.50 & 67.27 & NS & 33.93 \\
\hline $\mathrm{S}_{1}$ & 1530.30 & 866.44 & 1280.54 & 1292.00 & 587.91 & 720.99 & 357.84 & 372.26 \\
\hline $\mathrm{S}_{2}$ & 1480.56 & 885.40 & 1237.37 & 1200.74 & 1097.48 & 1406.61 & 898.60 & 715.61 \\
\hline $\mathrm{S}_{3}$ & 1426.58 & 960.33 & 1141.59 & 1158.12 & 1032.49 & 1556.63 & 1018.41 & 747.02 \\
\hline $\mathrm{S}_{4}$ & 1318.78 & 889.53 & 1026.68 & 1072.06 & 1120.54 & 1446.77 & 929.67 & 741.94 \\
\hline $\mathrm{S}_{5}$ & 1105.45 & 956.43 & 1135.06 & 1151.85 & 1212.98 & 1469.72 & 934.21 & 673.08 \\
\hline Mean & 1372.33 & 911.63 & 1164.25 & 1175.03 & 1010.28 & 1320.14 & 827.75 & 649.98 \\
\hline S. Em \pm & 73.99 & 27.40 & 18.94 & 28.36 & 32.73 & 35.80 & 33.53 & 18.06 \\
\hline C.D. at $5 \%$ & 219.81 & NS & 56.27 & 84.27 & 97.23 & 106.37 & 99.09 & 53.65 \\
\hline $\mathrm{C}_{1 \times} \mathrm{S}_{1}$ & 1648.37 & 877.87 & 1276.17 & 1297.59 & 598.92 & 602.71 & 328.60 & 342.96 \\
\hline $\mathrm{C}_{1 \times} \mathrm{S}_{2}$ & 1544.94 & 930.75 & 1259.30 & 1199.64 & 1117.95 & 1258.87 & 877.82 & 529.80 \\
\hline $\mathrm{C}_{1 \times} \mathrm{S}_{3}$ & 1418.90 & 999.75 & 1262.69 & 1232.44 & 1182.54 & 1474.45 & 1037.10 & 546.47 \\
\hline $\mathrm{C}_{1 \times} \mathrm{S}_{4}$ & 1294.05 & 911.37 & 1144.96 & 1158.31 & 1117.38 & 1356.72 & 889.90 & 565.48 \\
\hline $\mathrm{C}_{1 \times} \mathrm{S}_{5}$ & 777.14 & 1009.49 & 1237.08 & 1228.21 & 1208.04 & 1242.42 & 873.61 & 418.13 \\
\hline $\mathrm{C}_{2 \times} \mathrm{S}_{1}$ & 1412.22 & 848.35 & 1284.91 & 1286.41 & 576.92 & 839.27 & 387.08 & 404.56 \\
\hline $\mathrm{C}_{2 \times} \mathrm{S}_{2}$ & 1416.17 & 840.05 & 1215.44 & 1201.85 & 1077.01 & 1554.35 & 919.38 & 901.43 \\
\hline $\mathrm{C}_{2 \times} \mathrm{S}_{3}$ & 1434.25 & 920.92 & 1020.49 & 1084.58 & 882.43 & 1638.81 & 999.72 & 947.57 \\
\hline $\mathrm{C}_{2 \times} \mathrm{S}_{4}$ & 1343.52 & 867.69 & 908.40 & 985.81 & 1123.69 & 1536.82 & 969.45 & 918.40 \\
\hline $\mathrm{C}_{2 \times} \mathrm{S}_{5}$ & 1433.77 & 903.37 & 1033.05 & 1075.49 & 1217.92 & 1697.01 & 994.81 & 928.03 \\
\hline Mean & 1372.33 & 911.63 & 1164.25 & 1175.03 & 1010.28 & 1320.14 & 827.75 & 649.98 \\
\hline S. Em \pm & 104.64 & 38.75 & 26.79 & 40.11 & 46.29 & 50.64 & 47.17 & 25.54 \\
\hline C.D. at $5 \%$ & 310.86 & NS & 78.58 & NS & 137.51 & NS & NS & 75.87 \\
\hline CV \% & 13.20 & 7.36 & 3.98 & 5.91 & 7.93 & 6.64 & 9.87 & 6.81 \\
\hline
\end{tabular}


J. B. Patel et al. / J. Appl. \& Nat. Sci. 9 (1): 245- 252 (2017)

Table 6. Effects of storage condition and various seed treatments on seed vigour index-II in soybean.

\begin{tabular}{|c|c|c|c|c|c|c|c|c|}
\hline Factor/Period & 3 Month & 6 Month & 9 Month & 12 Month & 15 Month & 18 Month & 21 Month & 24 Month \\
\hline $\mathrm{C}_{1}$ & 847.45 & 910.43 & 925.44 & 762.09 & 758.23 & 797.33 & 377.65 & 214.73 \\
\hline $\mathrm{C}_{2}$ & 834.92 & 894.33 & 973.40 & 773.43 & 850.71 & 802.84 & 382.19 & 331.09 \\
\hline Mean & 841.18 & 902.38 & 949.42 & 767.76 & 804.47 & 800.08 & 379.92 & 272.91 \\
\hline $\mathrm{S} . \mathrm{Em} \pm$ & 11.60 & 13.58 & 11.12 & 15.46 & 16.43 & 10.03 & 4.39 & 3.25 \\
\hline C.D. at $5 \%$ & NS & NS & 33.05 & NS & 34.54 & NS & NS & 9.64 \\
\hline $\mathrm{S}_{1}$ & 852.24 & 837.89 & 924.17 & 818.80 & 688.76 & 654.52 & 290.68 & 161.96 \\
\hline $\mathrm{S}_{2}$ & 846.00 & 878.58 & 962.59 & 790.92 & 797.21 & 802.68 & 413.26 & 320.90 \\
\hline $\mathrm{S}_{3}$ & 860.79 & 954.45 & 992.12 & 769.02 & 836.05 & 916.32 & 404.85 & 307.11 \\
\hline $\mathrm{S}_{4}$ & 788.33 & 905.00 & 900.43 & 729.34 & 850.28 & 840.37 & 404.42 & 301.07 \\
\hline $\mathrm{S}_{5}$ & 858.56 & 935.96 & 967.78 & 730.74 & 850.05 & 786.51 & 386.37 & 273.52 \\
\hline Mean & 841.18 & 902.38 & 949.42 & 767.76 & 804.47 & 800.08 & 379.92 & 272.91 \\
\hline $\mathrm{S} . \mathrm{Em} \pm$ & 18.35 & 21.48 & 17.59 & 24.45 & 18.37 & 15.85 & 6.94 & 5.13 \\
\hline C.D. at $5 \%$ & NS & 63.83 & 52.26 & NS & 54.58 & 47.10 & 20.62 & 15.25 \\
\hline $\mathrm{C}_{1 \times} \mathrm{S}_{1}$ & 835.97 & 852.06 & 952.85 & 819.50 & 650.88 & 672.69 & 271.21 & 152.55 \\
\hline $\mathrm{C}_{1 \mathrm{X}} \mathrm{S}_{2}$ & 783.93 & 853.63 & 921.42 & 798.61 & 758.71 & 794.84 & 416.77 & 253.51 \\
\hline $\mathrm{C}_{1 \times} \mathrm{S}_{3}$ & 906.35 & 1010.79 & 1011.17 & 758.55 & 780.41 & 961.30 & 397.88 & 231.71 \\
\hline $\mathrm{C}_{1 \times} \mathrm{S}_{4}$ & 788.68 & 899.51 & 810.80 & 729.25 & 745.90 & 871.24 & 388.35 & 231.90 \\
\hline $\mathrm{C}_{1 \times} \mathrm{S}_{5}$ & 859.66 & 936.18 & 930.99 & 704.53 & 855.24 & 686.57 & 414.03 & 204.00 \\
\hline $\mathrm{C}_{2 \times} \mathrm{S}_{1}$ & 868.50 & 823.71 & 895.48 & 818.09 & 726.64 & 636.36 & 310.15 & 171.36 \\
\hline $\mathrm{C}_{2 \times} \mathrm{S}_{2}$ & 908.07 & 903.53 & 1003.76 & 783.22 & 835.70 & 810.52 & 409.75 & 388.30 \\
\hline $\mathrm{C}_{2 \times} \mathrm{S}_{3}$ & 815.22 & 898.15 & 973.10 & 779.48 & 891.69 & 871.33 & 411.81 & 382.52 \\
\hline $\mathrm{C}_{2 \times} \mathrm{S}_{4}$ & 787.98 & 910.50 & 990.07 & 729.43 & 954.67 & 809.51 & 420.49 & 370.26 \\
\hline $\mathrm{C}_{2 \times} \mathrm{S}_{5}$ & 857.47 & 935.76 & 1004.60 & 756.94 & 844.87 & 886.46 & 358.72 & 343.03 \\
\hline Mean & 841.18 & 902.38 & 949.42 & 767.76 & 804.47 & 800.08 & 379.92 & 272.91 \\
\hline $\mathrm{S} . \mathrm{Em} \pm$ & 25.95 & 30.38 & 52.26 & 34.57 & 25.98 & 22.42 & 9.81 & 7.26 \\
\hline C.D. at $5 \%$ & 77.10 & NS & 73.91 & NS & 77.18 & 66.61 & 29.16 & 21.56 \\
\hline $\mathrm{CV} \%$ & 5.34 & 5.83 & 4.53 & 7.79 & 5.59 & 4.85 & 4.47 & 4.61 \\
\hline
\end{tabular}

Table 7. Effects of storage condition and various seed treatments on seed moisture in soybean.

\begin{tabular}{|c|c|c|c|c|c|c|c|c|}
\hline Factor/Period & 3 Month & 6 Month & 9 Month & 12 Month & 15 Month & 18 Month & 21 Month & 24 Month \\
\hline $\mathrm{C}_{1}$ & 3.37 & 4.87 & 5.97 & 5.61 & 5.31 & 6.07 & 7.02 & 7.19 \\
\hline $\mathrm{C}_{2}$ & 3.44 & 4.31 & 4.72 & 4.76 & 4.93 & 5.31 & 5.29 & 5.49 \\
\hline Mean & 3.40 & 4.59 & 5.35 & 5.19 & 5.12 & 5.69 & 6.15 & 6.34 \\
\hline $\mathrm{S} . \mathrm{Em} \pm$ & 0.02 & 0.04 & 0.02 & 0.04 & 0.09 & 0.04 & 0.03 & 0.07 \\
\hline C.D. at $5 \%$ & NS & 0.13 & 0.06 & 0.11 & 0.27 & 0.11 & 0.08 & 0.21 \\
\hline $\mathrm{S}_{1}$ & 3.48 & 4.48 & 5.41 & 5.34 & 4.90 & 5.65 & 6.17 & 6.29 \\
\hline $\mathrm{S}_{2}$ & 3.45 & 4.71 & 5.53 & 5.15 & 4.91 & 5.57 & 6.37 & 6.55 \\
\hline $\mathrm{S}_{3}$ & 3.50 & 4.75 & 5.32 & 5.34 & 5.16 & 5.89 & 6.07 & 6.20 \\
\hline $\mathrm{S}_{4}$ & 3.58 & 4.73 & 5.43 & 5.31 & 5.64 & 5.55 & 6.10 & 6.35 \\
\hline $\mathrm{S}_{5}$ & 3.01 & 4.30 & 5.04 & 4.79 & 4.99 & 5.80 & 6.06 & 6.30 \\
\hline Mean & 3.40 & 4.59 & 5.35 & 5.19 & 5.12 & 5.69 & 6.15 & 6.34 \\
\hline S. Em \pm & 0.04 & 0.07 & 0.03 & 0.06 & 0.15 & 0.06 & 0.04 & 0.11 \\
\hline C.D. at $5 \%$ & 0.11 & 0.20 & 0.10 & 0.18 & 0.43 & 0.17 & 0.13 & NS \\
\hline $\mathrm{C}_{1 \times} \mathrm{S}_{1}$ & 3.51 & 4.67 & 5.95 & 5.69 & 5.35 & 5.95 & 6.88 & 7.07 \\
\hline $\mathrm{C}_{1 \mathrm{X}} \mathrm{S}_{2}$ & 3.38 & 5.04 & 6.03 & 5.81 & 4.45 & 6.09 & 7.40 & 7.56 \\
\hline $\mathrm{C}_{1 \times} \mathrm{S}_{3}$ & 3.44 & 4.84 & 5.86 & 5.50 & 5.60 & 6.40 & 6.94 & 7.10 \\
\hline $\mathrm{C}_{1 \mathrm{X}} \mathrm{S}_{4}$ & 3.43 & 5.30 & 6.13 & 5.73 & 5.83 & 5.81 & 6.90 & 7.20 \\
\hline $\mathrm{C}_{1 \mathrm{X}} \mathrm{S}_{5}$ & 3.08 & 4.51 & 5.70 & 5.31 & 5.30 & 6.10 & 6.97 & 7.07 \\
\hline $\mathrm{C}_{2 \times} \mathrm{S}_{1}$ & 3.45 & 4.28 & 4.88 & 4.99 & 4.44 & 5.34 & 5.45 & 5.53 \\
\hline $\mathrm{C}_{2 \times} \mathrm{S}_{2}$ & 3.52 & 4.37 & 4.83 & 4.49 & 5.37 & 5.04 & 5.34 & 5.53 \\
\hline $\mathrm{C}_{2 \times} \mathrm{S}_{3}$ & 3.55 & 4.67 & 4.78 & 5.18 & 4.72 & 5.38 & 5.21 & 5.33 \\
\hline $\mathrm{C}_{2 \times} \mathrm{S}_{4}$ & 3.73 & 4.16 & 4.73 & 4.90 & 5.45 & 3.30 & 5.31 & 5.51 \\
\hline $\mathrm{C}_{2 \times} \mathrm{S}_{5}$ & 2.94 & 4.09 & 4.38 & 4.25 & 4.68 & 5.49 & 5.15 & 5.53 \\
\hline Mean & 3.40 & 4.59 & 5.35 & 5.19 & 5.12 & 5.69 & 6.15 & 6.34 \\
\hline $\mathrm{S} . \mathrm{Em} \pm$ & 0.05 & 0.10 & 0.05 & 0.08 & 0.21 & 0.08 & 0.06 & 0.16 \\
\hline C.D. at $5 \%$ & 0.16 & 0.29 & 0.14 & 0.25 & 0.61 & 0.25 & 0.18 & NS \\
\hline $\mathrm{CV} \%$ & 2.65 & 3.61 & 1.54 & 2.80 & 6.93 & 2.53 & 1.74 & 4.39 \\
\hline
\end{tabular}


Table 8. Germination percentage recorded in soybean seed after bringing out the sample stored under cold storage condition $\left(7^{0} \mathrm{C} \pm 2^{0} \mathrm{C}\right)$ packed with cloth bag.

\begin{tabular}{lcccccc}
$\begin{array}{l}\text { Days after sample } \\
\text { bringing out from cold } \\
\text { storage condition }\end{array}$ & $\begin{array}{c}\text { DATE of } \\
\text { sampling }\end{array}$ & $\begin{array}{c}\text { Date of re- } \\
\text { cording } \\
\text { germination }\end{array}$ & $\begin{array}{c}\text { Mencozeb @ } \\
\text { 2g/kg seed }\end{array}$ & $\begin{array}{c}\text { Carbendazim } \\
\text { a } \mathbf{2 g} / \mathbf{k g} \text { seed }\end{array}$ & $\begin{array}{c}\text { Neam Leaf } \\
\text { powder @ 10g/ } \\
\text { kg seed }\end{array}$ & $\begin{array}{c}\text { Neem Oil } \\
\text { @ 5 } \mathbf{~ m l / k g ~} \\
\text { seed }\end{array}$ \\
\hline 0 & $12-10-2015$ & $20-10-2015$ & 83 & 87 & 84 & 83 \\
2 & $14-10-2015$ & $22-10-2015$ & 83 & 85 & 84 & 81 \\
3 & $15-10-2015$ & $23-10-2015$ & 71 & 82 & 73 & 80 \\
5 & $17-10-2015$ & $26-10-2015$ & 76 & 86 & 80 & 85 \\
7 & $19-10-2015$ & $27-10-2015$ & 75 & 83 & 84 & 84 \\
9 & $21-10-2015$ & $29-10-2015$ & 84 & 79 & 79 & 80 \\
11 & $23-10-2015$ & $01-11-2015$ & 82 & 77 & 79 & 83 \\
14 & $26-10-2015$ & $03-11-2015$ & 81 & 80 & 79 & 80 \\
16 & $28-10-2015$ & $05-11-2015$ & 80 & 80 & 79 & 79 \\
18 & $30-10-2015$ & $07-11-2015$ & 69 & 78 & 79 & 77 \\
20 & $02-11-2015$ & $09-11-2015$ & 68 & 71 & 66 & 70 \\
22 & $04-11-2015$ & $12-11-2015$ & 83 & 66 & 67 & 81 \\
24 & $06-11-2015$ & $14-11-2015$ & 73 & 77 & 80 & 71 \\
25 & $07-11-2015$ & $16-11-2015$ & 72 & 74 & 72 & 73 \\
38 & $20-11-2015$ & $28-11-2015$ & 72 & 68 & 77 & 72 \\
45 & $27-11-2015$ & $05-12-2015$ & 69 & 70 & 73 & 68 \\
53 & $05-12-2015$ & $14-12-2015$ & 66 & 68 & 70 & 67 \\
64 & $16-12-2015$ & $24-12-2015$ & 73 & 66 & 73 & 63 \\
\hline
\end{tabular}

combinations, more than 80 per cent germination was recorded by the combination of seed treated with fungicide and stored in cold storage after two years of storage. These results are in accordance with the results of Gupta et al. (1976), who reported that soybean seeds are short lived as compared to maize, rice, wheat, etc. The soybean seeds having only high initial germination $(>80-90 \%)$ could be recommended for one season storage. Storing soybean seeds beyond first planting season at room temperature may not be successful even in moisture resistant containers. Upto second planting season soybean could be safely stored in cold storage $\left(4-5^{\circ} \mathrm{C}\right.$ temperature and $50-60 \%$ relative humidity). Mbofung et al. (2013) reported that treated soybean seeds could be carried over for two seasons, if the storage temperature is maintained at $10^{\circ}$ $\mathrm{C}$ and the relative humidity is below 40 per cent.

The results presented in Table 2 revealed that storage condition (C) and seed treatments (S) exhibited significant differences for seedling root length in soybean for most of the dates. Over all the treatment combinations showed non-significant difference with respect to root length after 2 years of storage. The results presented in Table 3 revealed that storage condition (C) and seed treatments (S) exhibited significant differences for seedling shoot length in soybean after 2 years of storage. The data of soybean seed stored under two storage conditions revealed that seed stored under cold storage $\left(7^{\circ} \mathrm{C} \pm 2^{\circ} \mathrm{C}\right)$ noted significantly higher seedling shoot length $(5.37 \mathrm{~cm})$ after the period of 2 years of storage. Among the seed treatments, seed treated with Neem leaf powder@10g/kg seed recorded the significantly highest seedling shoot length $(5.23 \mathrm{~cm})$ after 2 years of storage. Over all the treatment combinations, $\mathrm{C}_{2} \times \mathrm{S}_{5}$ recorded the highest value for shoot length $(6.10 \mathrm{~cm})$.
The results presented in Table 4 revealed that seed treatments (S) exhibited significant differences for seedling dry weight in soybean after 2 years of storage, while storage condition (C) exhibited non-significant for this trait after 2 years of storage. Among the seed treatments, seed treated with Carbendazim @ 2g/kg seed recorded the significantly highest seedling dry weight $(4.62 \mathrm{~g})$ after 2 years of storage. Over all the treatment combinations, $\mathrm{C}_{1 \times} \mathrm{S}_{2}$ recorded the highest value for seedling dry weight $(4.69 \mathrm{~g})$.

The results presented in Table 5 revealed that storage condition (C) and seed treatments (S) exhibited significant differences for seed vigour index $\mathrm{I}$ in soybean after 2 years of storage. The data of soybean seed stored under two storage conditions revealed that seed stored under cold storage $\left(7^{\circ} \mathrm{C}+2^{\circ} \mathrm{C}\right)$ noted significantly higher seed vigour index I (819.40) after the period of 2 years of storage. Among the seed treatments, seed treated with Mancozeb @ 2g/kg of seed recorded the significantly highest seed vigour index I (747.02) and it was at par with Neem leaf powder@10g/kg seed (741.94) and Carbendazim @ $2 \mathrm{~g} / \mathrm{kg}$ seed (715.61) after 2 years of storage. Seed vigour index I noted in control treatment was 372.26 after 2 years of storage. Over all the treatment combinations, more than 900 seed vigour index was recorded by the combination of seed treated with fungicide and stored in cold storage after two years of storage. The results presented in Table 6 revealed that storage condition (C) and seed treatments (S) exhibited significant differences for seed vigour index II in soybean after 2 years of storage. The data of soybean seed stored under two storage conditions revealed that seed stored under cold storage $\left(7^{\circ} \mathrm{C} \pm 2^{\circ} \mathrm{C}\right)$ noted significantly higher seed vigour index II $(331.09)$ after 
the period of 2 years of storage. Among the seed treatments, seed treated with Carbendazim @ $2 \mathrm{~g} / \mathrm{kg}$ of seed recorded the significantly highest seed vigour index II (320.11) and it was at par with mancozeb @ $2 \mathrm{~g} / \mathrm{kg}$ seed (307.11) after 2 years of storage. Seed vigour index II noted in control treatment was 161.96 after 2 years of storage. Over all the treatment combinations, more than 200 seed vigour index was recorded by the combination of seed treated with fungicide and stored in cold storage after two years of storage.

The results presented in Table 7 revealed that storage condition (C) and exhibited significant differences for seed moisture content after 2 years of storage, however, seed treatments (S) and treatment combinations $\left(\mathrm{C}_{\mathrm{X}} \mathrm{S}\right)$ were non-significant after two years of storage. The results of soybean seed stored in two storage conditions showed that on an average, the seed stored in ambient storage condition manifested significantly higher seed moisture content.

The decline in germination percentage may be attributed to ageing effect leading to depletion of food reserves and decline in synthetic activity of embryo apart from death of seed because of fungal invasion, insect damage, fluctuating temperature, relative humidity. Similar results were reported by Vidhyasekaran et al. (1980) in sorghum and millet, Ashokan et al. (1981) in finger millet, Hooda and Singh (1993) in wheat and Anitha et al. (2013) in soybean.

The variation in germination percentage of soybean seed decreased with increase storage period which might be due to the deleterious effects of moisture which resulted from the storage materials and perhaps environmental conditions. This agrees with Tame (2011) who reported that germination percentage of soybean seed decreased with increase in storage period. Arif et al. (2006) recorded that highest germination percentages in soybean were in seed stored at $4^{\circ} \mathrm{C}$ than room temperature. Kandil et al. (2013) observed maximum seed germination parameters in soybean, when the seeds of Giza 111 cultivar stored under refrigerator conditions $\left(10 \pm 1^{\circ} \mathrm{C}\right)$ in cloth bags for 3 months.

In recent years however, attempts have been made to replace synthetic (inorganic) seed treatment chemicals with organic materials of plant origin which are cheaper, safer and eco-friendly. Among the various methods followed, use of botanicals has been a traditional method and is being given much attention. Arati (2000) reported that Bengalgram seeds treated with neem leaf powder recorded higher germination (65.91\%) and vigour index (1282) compared to control at the end of 10 months of storage period. Maraddi (2002) observed cowpea seeds treated with neem leaf powder $(5 \mathrm{~g} / \mathrm{kg})$ recorded higher germination $(39.5 \%)$ and vigour index (1072) compared to control $(34.2 \%$ and 864 , respectively) at the end of 10 months of storage period. Oyekole et al. (2012) reported that seed treatment with neem leaf powder is specifically recommended for pretreatment storage of sesame seeds, as it maintained seed viability and seedling vigour optimally among all other treatments.

\section{Conclusion}

It can be concluded that soybean seed may be stored under cold storage $\left(7^{\circ} \mathrm{C} \pm 2^{\circ} \mathrm{C}\right)$ condition in muslin cloth bag with seed treatment of Mancozeb@2g/kg seed or Carbendazim @ $2 \mathrm{~g} / \mathrm{kg}$ seed or Neem leaf powder@10 g / kg seed for a period of 2 years without deterioration in germination and seedling vigour.

\section{REFERENCES}

Abdul-Baki, A.A. and Anderson, J. D. (1973). Vigor determination in soybean seeds by multiple criteria. Crop Sci., 13: 630-633

Anitha, Mummigatti, U. V., Madhusudhan and Punith Kumar, C. H. (2013). Effect of orbanic and inorganic seed priming on soybean germination and yield parameters. Biolife, 1(4): 223-230

Arati, P. (2000). Influence of containers and seed treatment on storability of chickpea. M.Sc. (Agric.) Thesis. University of Agricultural Sciences, Dharwad. pp. 255.

Arif, M. (2006). Effect of seed priming on emergence, yield and storability of soybean. Ph.D Dissertation, NWFP Agricultural University Peshawar, Pakistan.

Ashokan, A., Emayavaramban, N. and Ramabadran, R. (1981). Effect of fungicidal treatments on viability of finger millet seeds during storage. Seed Res.,9(2):90-91

Cochran, V. G. and Cox, G. M. (1957). Experimental Designs. New York, John Wiley and Hall Ltd., London, pp. $293 \cdot 296$

Fabrizius, E., TeKrony, D., Egli, D. B. and Rucker, M. (1999). Evaluation of a viability model for predicting soybean seed germination during warehouse storage. Crop Sci., 39: 194-201

Gupta, S. P., Madan, P. S. and Kaur, J. (1976). Effect of seed size on seed yield and seed quality in wheat. Seeds Farms, 13: 11-13

Hooda, K. S. and Singh, M. (1993). Storage of vitavax treated wheat seeds in relation to seed moisture and control of loose smut in field. Seed Res., 21(2): 123-125

ISTA (1999). International rules for seed testing. Seed Sci. Tahnol., Supplement Rules, 27: 20-25

Kandil, A. A., Sharief, A. E. and Sheteiwy, M. S. (2013). Effect of seed storage periods, conditions and materials on germination of some soybean seed cultivars. American J. Exptl. Agric., 3(4): 1020-1043

Mahesh Babu, H. M. and Hunje, R. (2008). Effect of seed treatment with botanicals on storability of soybean. Karnataka J. Agric. Sci., 21(3): 357-360

Maraddi, B. (2002). Influence of growth regulators on seed yield and quality and seed treatments on storability of cowpea cv. C-252, M.Sc. (Agri.) Thesis. University of Agricultural Sciences, Dharwad. pp. 216.

Mbofung, G. C. Y., Goggi, A. S., Leandro, L. F. S. and Mullen, R.E. (2013). Effects of storage temperature and relative humidity on viability and vigor of treated soybean seeds. Crop Sci., 53: 1086-1095 
Oyekole, K.O., Nwangburuka, C.C., Denton, O.A., Daramola, D.S., Adeyeye, J. A. and Akinkuotu, A.O. (2012). Comparative effects of organic and inorganic seed treatments on the viability and vigour of sesame seeds in storage. J. Agril. Sci., 4(9): 187-196

Sisman, C. (2005). Quality losses in temporary sunflower stores and influences of storage conditions on quality losses during storage. J. Central European Agric., 6:
$143-150$

Tame, V.T. (2011). Viability and vigour of soybean seed (Glycine $\max$ (L.) Merr.). LAP Lambert Academic Publishing $\mathrm{GmbH} \& \mathrm{Co}$. KG, Germany.

Vidhyasekaran, P., Thulasidas, G., Ramaswamy, K. R. and Kandaswamy, T. K. (1980). Presevation of viability of sorghum seeds by controlling seed-borne fungi. Indian Phytopath., 33(2):225-230 